\title{
Monte Carlo modeling and simulations of the High Definition (HD120) micro MLC and validation against measurements for a $6 \mathrm{MV}$ beam
}

$\underline{\text { C. Borges }}^{1, a)}, \underline{\text { M. Zarza-Moreno }}{ }^{2}, \underline{\text { E. Heath }}^{3}, \underline{\text { N. Teixeira }}^{4}$ and $\underline{\text { P. Vaz }}{ }^{5}$ +View Affiliations

a) Author to whom correspondence should be addressed. Electronic mail: cissa9@gmail.com

Med. Phys. 39, 415 (2012); http://dx.doi.org/10.1118/1.3671935

- $\quad$ Previous Article

- Table of Contents

- Next Article

- Abstract

- Full Text

- References (36)

- Cited By

- Data \& Media

- Metrics

- $\quad$ Related

\section{Purpose:}

The most recent Varian ${ }^{\circledR}$ micro multileaf collimator(MLC), the High Definition (HD120) MLC, was modeled using the BEAMNRCMonte Carlo code. This model was incorporated into a Varian medical linear accelerator, for a $6 \mathrm{MV}$ beam, in static and dynamic mode. The model was validated by comparing simulated profiles with measurements.

\section{Methods:}

The Varian ${ }^{\oplus}$ Trilogy ${ }^{\oplus}$ (2300C/D) accelerator model was accurately implemented using the state-of-the-art Monte Carlo simulation program BEAMNRC and validated against off-axis and depth dose profiles measured using ionization chambers, by adjusting the energy and the full width at half maximum (FWHM) of the initial electron beam. The HD120 MLC was modeled by developing a new BEAMNRC component module (CM), designated HDMLC, adapting the available DYNVMLC CM and incorporating the specific characteristics of this new micro MLC. The leaf dimensions were provided by the manufacturer. The geometry was visualized by tracing particles through the CM and recording their position when a leaf boundary is crossed. The leaf material density and abutting air gap between leaves were adjusted in order to obtain a good agreement between the simulated leakage profiles and EBT2 film measurements performed in a solid water phantom. To validate the HDMLC implementation, additional MLC static patterns were also simulated and compared to additional measurements. Furthermore, the ability to simulate dynamic MLC fields was implemented in the HDMLC CM. The simulation results of these fields were compared with EBT2 film measurements performed in a solid water phantom. 


\section{Results:}

Overall, the discrepancies, with and without MLC, between the opened field simulations and the measurements using ionization chambers in a water phantom, for the off-axis profiles are below $2 \%$ and in depth-dose profiles are below $2 \%$ after the maximum dose depth and below $4 \%$ in the build-up region. On the conditions of these simulations, this tungsten-based MLC has a density of $18.7 \mathrm{~g} \mathrm{~cm}^{-3}$ and an overall leakage of about $1.1 \pm 0.03 \%$. The discrepancies between the film measured and simulated closed and blocked fields are below $2 \%$ and $8 \%$, respectively. Other measurements were performed for alternated leaf patterns and the agreement is satisfactory (to within 4\%). The dynamic mode for this MLC was implemented and the discrepancies between film measurements and simulations are within $4 \%$.

\section{Conclusions:}

The Varian ${ }^{\oplus}$ Trilogy $^{\oplus}(2300$ C/D) linear accelerator including the HD120 MLC was successfully modeled and simulated using the Monte CarloBEAMNRC code by developing an independent CM, the HDMLC CM, either in static and dynamic modes.

(C) 2012 American Association of Physicists in Medicine

Received 23 August 2011 Revised 23 November 2011 Accepted 01 December 2011 Published online 27 December 2011

Acknowledgments:

Varian Medical Systems (Palo Alto, CA) are sincerely acknowledged for having provided all the specifications needed for the linac simulations. The authors express their thanks to Quadrantes, Centro Oncológico Dra. Natália Chaves (Portugal) for providing the logistic resources.

Article outline:

I. INTRODUCTION

II. MATERIALS AND METHODS

II.A. Monte Carlo simulations

II.A.1. Accelerator up to the jaws

II.A.2. Accelerator including the HD120 MLC

II.B. Measurements

III. RESULTS AND DISCUSSION

III.A. Accelerator up to the jaws

III.B. Including the HD120 MLC

III.B.1. Ray tracing

III.B.2. Tuning of the MLC: assessment of the density of the leaves and of the abutting gap

III.B.3. Irregular MLC pattern

III.B.4. Ionometric measurements

III.B.5. Dynamic mode

IV. CONCLUSIONS 